\title{
Probabilistic Verification of Multiple-Valued Functions
}

\author{
Elena Dubrova \\ Department of Electronics \\ Royal Institute of Technology \\ S-164 40 Kista \\ Sweden \\ elena@ele.kth.se
}

\author{
Harald Sack \\ FB IV - Informatik \\ Universität Trier \\ D-54286 Trier \\ Germany \\ sack@uni-trier.de
}

\begin{abstract}
This paper describes a probabilistic method for verifying the equivalence of two multiple-valued functions. Each function is hashed to an integer code by transforming it to a integer-valued polynomial and the equivalence of two polynomials is checked probabilistically. The hash codes for two equivalent functions are always the same. Thus, the equivalence of two functions can be verified with a known probability of error, arising from collisions between inequivalent functions. Such a probabilistic verification can be an attractive alternative for verifying functions that are too large to be handled by deterministic verification methods.
\end{abstract}

\section{Introduction}

In recent years, advances in integrated circuit technology made feasible fabrication of several commercial products benefiting from multiple-valued logic, such as $256-$ Mbit 4valued flash memory [1] and 4-Gbit 4-valued DRAM [2]. These products can be seen as first steps toward recognition of the increasing role of multiple-valued logic in the next generation of electronic systems. However, for further practical utilization, efficient computer-aided tools for design, testing and verification of multiple-valued logic circuits are needed. Some existing tools, such as Berkeley's tool for verification and synthesis VIS [3], provide a solution for the special case of multiple-valued input binary-valued output functions, but the general problem is still open.

This paper focuses on the problem of verification of multiple-valued functions. Up to now, very little research is done in this area. Some techniques for verification of Boolean circuits, such as random simulation or symbolic simulation, can be directly applied to verification of multiple-valued logic case [4], [5]. Similarly, verification procedures employing Reduced Ordered Binary Decision
Diagrams (ROBDDs) [6] can adapted to Multiple-valued Decision Diagrams (MDD) [7] as shown in [8]. However, the MDD verification methods representing functions as single, monolithic graph might be infeasible for large functions. We believe that deterministic methods of verification will not be practical for the multiple-valued logic domain due to the increasing complexity of the problem.

In this paper we present a probabilistic method for design verification of multiple-valued logic functions, which generalizes the method introduced in [9]. We define a functional transformation $A$ which converts a multiple-valued function $f: M^{n} \rightarrow M$ on a set $M \stackrel{d f}{=}\{0,1, \ldots, m-1\}$ into a polynomial of type $A_{m}[f]: Z_{p}^{n} \rightarrow Z_{p}$ over a finite field of integers $Z_{p}$ modulo $p$, for some prime $p$. This polynomial is used to generate a hash code for $f$, by evaluating the value of $A_{m}[f]\left(x_{1}, \ldots, x_{n}\right)$ for randomly chosen values of $x_{i}$ from $Z_{p}, i \in\{1, \ldots, n\}$. The hash codes for two equivalent functions are always the same. Thus, the equivalence of two functions can be verified with a known probability of error, arising from collisions between inequivalent functions.

The paper is organized as follows. In Section 1, the $A$ transform is defined and its properties are studied. Section 2 shows how to compute the integer-valued polynomial given by $A$-transform. Section 3 defines the notion of hash code for a function. In Section 4, we give a conclusion and describe the topics for further research.

\section{Definition and properties of $A$-transform}

To define the transformation $A$, we associate a key polynomial with each of the $m^{n}$ input assignments of a multiplevalued function $f\left(x_{1}, \ldots, x_{n}\right)$. We then sum up the key polynomials of assignments producing the non-zero output value of $f$, and interpret the result as a integer-valued function $A_{m}[f]\left(x_{1}, \ldots, x_{n}\right)$ over $Z_{p}$.

The key polynomial for a given row of the truth table is a 
product of terms, where each term is associated with a particular input variable $x_{i}, i \in\{1, \ldots, n\}$. If $b_{i}$ represents the value of $x_{i}$ in a given row of the truth table, then the corresponding term $w\left(b_{i}, x_{i}\right)$ in the key polynomial is defined as follows:

Definition 1 For any $m>1, w: Z_{p} \times Z_{p} \rightarrow Z_{p}$ is defined by

$$
w(b, x)=\sum_{i=0}^{m-1}\left(\prod_{j \in M-\{i\}} \frac{j-b}{j-i} \cdot \prod_{j \in M-\{i\}} \frac{j-x}{j-i}\right)
$$

It is easy to see that $\prod_{j \in M-\{i\}} \frac{j-b}{j-i}=1$ for $b=i$ and $\prod_{j \in M-\{i\}} \frac{j-b}{j-i}=0$ for $b \neq i$. Therefore, parameter $b$ acts as a selector between the terms $\prod_{j \in M-\{\}\}} \frac{j-x}{j-i}$ for different values of $i \in M$, i.e. $w(0, x)=\prod_{j \in M-\{0\}} \frac{j-x}{j}$, $w(1, x)=\prod_{j \in M-\{1\}} \frac{j-x}{j-1}$, and so on. On the other hand, each of the terms $\prod_{j \in M-\{i\}} \frac{j-x}{j-i}$, represents a polynomial which evaluates to 1 for $x=i$ and evaluates to 0 for $x \in M-\{i\}$. So, such a polynomial has a behavior similar to the behavior of the literal operator $\stackrel{i}{x}$ :

$$
\stackrel{i}{x} \stackrel{d f}{=} \begin{cases}m-1 & \text { if } x=i \\ 0 & \text { otherwise }\end{cases}
$$

except that for $x=i$ the literal $\stackrel{i}{x}$ evaluates to $m-1$, and not to 1 .

The key polynomial $W_{n}$ for an assignment $\left(b_{1}, \ldots, b_{n}\right) \in$ $M^{n}$ of $n$ variables is defined as the product of the $w\left(b_{i}, x_{i}\right)$ terms, $i \in\{1, \ldots, n\}$ :

Definition 2 For any $n \geq 0$ and $m>1$, the key polynomial $W_{n}: Z_{p}^{2 n} \rightarrow Z_{p}$ is defined by

$$
W_{n}\left(b_{1}, \ldots, b_{n}, x_{1}, \ldots, x_{n}\right)=\prod_{i=1}^{n} w\left(b_{i}, x_{i}\right)
$$

For example, for $m=3$ :

$$
W_{2}\left(0,1, x_{1}, x_{2}\right)=\frac{1}{2}\left(1-x_{1}\right)\left(2-x_{1}\right) x_{2}\left(2-x_{2}\right) .
$$

Similarly:

$$
W_{2}\left(1,2, x_{1}, x_{2}\right)=x_{1}\left(2-x_{1}\right)\left(-\frac{1}{2} x_{2}\right)\left(1-x_{2}\right)
$$

Now, we define the transformation $A$ as a sum of key polynomials $W_{n}$ for all assignments $\left(b_{1}, \ldots, b_{n}\right) \in M^{n}$, each multiplied by the value of $f$ for the corresponding assignment. We give a definition, applicable for general functions $Z_{p}^{n} \rightarrow Z_{p}$ over the field $Z_{p}$. Note that, since $M \subset Z_{p}$, the multiple-valued functions $M^{n} \rightarrow M$ are a subset of the field functions $Z_{p}^{n} \rightarrow Z_{p}$. While a field function is defined for inputs $x_{i} \notin M$ as well, the values which the function produces for such assignments do not participate in the definition. To distinguish between multiple-valued and field functions, throughout the paper we use the unsubscribed letters $f, g$ for multiple-valued functions of type $M^{n} \rightarrow M$ and the subscribed letters $f_{z}, g_{z}$ for field functions of type $Z_{p}^{n} \rightarrow Z_{p}$.

Definition 3 Given a function of type $f_{z}: Z_{p}^{n} \rightarrow Z_{p}$ and $m>1$, the polynomial $A_{m}\left[f_{z}\right]: Z_{p}^{n} \rightarrow Z_{p}$ is defined by

$$
\begin{aligned}
& A_{m}\left[f_{z}\right]\left(x_{1}, \ldots, x_{n}\right)= \\
& =\sum_{\left(b_{1}, . ., b_{n}\right) \in M^{n}} f_{z}\left(b_{1}, . ., b_{n}\right) \cdot W_{n}\left(b_{1}, . ., b_{n}, x_{1}, \ldots, x_{n}\right)
\end{aligned}
$$

For example, for $m=3$ and $n=2$, the polynomial $A_{3}\left[f_{z}\right]\left(x_{1}, x_{2}\right)$ is given by

$$
\begin{aligned}
& A_{3}\left[f_{z}\right]\left(x_{1}, x_{2}\right)= \\
& \quad=f(0,0) \cdot \frac{1}{2}\left(1-x_{1}\right)\left(2-x_{1}\right)\left(1-x_{2}\right)\left(2-x_{2}\right)+ \\
& \quad+f(0,1) \cdot \frac{1}{2}\left(1-x_{1}\right)\left(2-x_{1}\right) x_{2}\left(2-x_{2}\right)+ \\
& \quad+f(0,2) \cdot \frac{1}{4}\left(1-x_{1}\right)\left(2-x_{1}\right)\left(-x_{2}\right)\left(1-x_{2}\right)+ \\
& \quad+f(1,0) \cdot x_{1}\left(2-x_{1}\right)\left(1-x_{2}\right)\left(2-x_{2}\right)+ \\
& \quad+f(1,1) \cdot x_{1}\left(2-x_{1}\right) x_{2}\left(2-x_{2}\right)+ \\
& \quad+f(1,2) \cdot x_{1}\left(2-x_{1}\right)\left(-x_{2}\right)\left(1-x_{2}\right)+ \\
& \quad+f(2,0) \cdot \frac{1}{2}\left(-x_{1}\right)\left(1-x_{1}\right)\left(1-x_{2}\right)\left(2-x_{2}\right)+ \\
& \quad+f(2,1) \cdot \frac{1}{2}\left(-x_{1}\right)\left(1-x_{1}\right) x_{2}\left(2-x_{2}\right)+ \\
& \quad+f(2,2) \cdot \frac{1}{4}\left(-x_{1}\right)\left(1-x_{1}\right)\left(-x_{2}\right)\left(1-x_{2}\right) .
\end{aligned}
$$

E.g. for the 3-valued function $f\left(x_{1}, x_{2}\right)=\operatorname{MIN}\left(x_{1}, x_{2}\right)$, the corresponding polynomial is

$A_{3}[f]\left(x_{1}, x_{2}\right)=x_{1}\left(2-x_{1}\right) x_{2}\left(2-x_{2}\right)+x_{1}(2-$ $\left.x_{1}\right)\left(-x_{2}\right)\left(1-x_{2}\right)+\left(-x_{1}\right)\left(1-x_{1}\right) x_{2}\left(2-x_{2}\right)+2\left(-x_{1}\right)(1-$ $\left.x_{1}\right)\left(-x_{2}\right)\left(1-x_{2}\right)=\frac{5}{2} x_{1} x_{2}-x_{1} x_{1}^{2}-x_{1}^{2} x_{2}+\frac{1}{2} x_{1}^{2} x_{2}^{2}$.

Note that the $A$-transform is defined only for assignments $\left(b_{1}, \ldots, b_{n}\right) \in M^{n}$. Therefore, if two field functions $f_{z}$ and $g_{z}$ have the same values for all $\left(b_{1}, \ldots, b_{n}\right) \in M^{n}$, then they are treated identically by the $A$-transform. We say that two such functions are $m$-equivalent:

Definition 4 The functions $f_{z}$ and $g_{z}$ of type $Z_{p}^{n} \rightarrow Z_{p}$ are $m$-equivalent if and only if $f_{z}\left(b_{1}, \ldots, b_{n}\right)=g_{z}\left(b_{1}, \ldots, b_{n}\right)$ for any assignment $\left(b_{1}, \ldots, b_{n}\right) \in M^{n}$. 
We write $f_{z} \stackrel{m}{=} g_{z}$ to denote that $f_{z}$ and $g_{z}$ are $m$-equivalent. If both $f$ and $g$ are multiple-valued functions of type $M^{n} \rightarrow$ $M$ then $f \stackrel{m}{=} g$ is the same as $f=g$.

By Definition 3, $f_{z} \stackrel{m}{=} g_{z}$ implies $A_{m}\left[f_{z}\right]=$ $A_{m}\left[f_{z}\right]$. However, can we conclude $A_{m}\left[f_{z}\right] \neq A_{m}\left[f_{z}\right]$ from $f_{z} \stackrel{m}{\neq} g_{z}$ ? To answer this question let us examine the behavior of polynomial $A_{m}[f]$ when it is evaluated for some assignment $\left(b_{1}, \ldots, b_{n}\right) \in M^{n}$. It is easy to see that for any $b, b^{\prime} \in M, w\left(b, b^{\prime}\right)=1$ if $b=b^{\prime}$, and $w\left(b, b^{\prime}\right)=0$ otherwise. Therefore, for any $\left(b_{1}, \ldots, b_{n}\right),\left(b_{1}^{\prime}, \ldots, b_{n}^{\prime}\right) \in$ $M^{n}, W\left(b_{1}, \ldots, b_{n}, b_{1}^{\prime}, \ldots, b_{n}^{\prime}\right)=1$ if $b_{i}=b_{i}^{\prime}$ for all $i \in\{1, \ldots, n\}$, and $W\left(b_{1}, \ldots, b_{n}, b_{1}^{\prime}, \ldots, b_{n}^{\prime}\right)=0$ otherwise. Using these facts, we can prove the following theorem:

Theorem 1 For any function $f_{z}: Z_{p}^{n} \rightarrow Z_{p}, A_{m}\left[f_{z}\right] \stackrel{m}{=} f_{z}$.

Proof: By Definition 3, for any $\left(b_{1}^{\prime}, \ldots, b_{n}^{\prime}\right) \in M^{n}$ we have:

$$
\begin{aligned}
& A_{m}\left[f_{z}\right]\left(b_{1}^{\prime}, \ldots, b_{n}^{\prime}\right)= \\
& =\sum_{\left(b_{1}, . ., b_{n}\right) \in M^{n}} f_{z}\left(b_{1}, \ldots, b_{n}\right) \cdot W_{n}\left(b_{1}, \ldots, b_{n}, b_{1}^{\prime}, \ldots, b_{n}^{\prime}\right)
\end{aligned}
$$

Since $W\left(b_{1}, \ldots, b_{n}, b_{1}^{\prime}, \ldots, b_{n}^{\prime}\right)=1$ only if $b_{i}=b_{i}^{\prime}$ for all $i \in\{1, \ldots, n\}$, and 0 otherwise, this gives us $A_{m}\left[f_{z}\right]\left(b_{1}^{\prime}, \ldots, b_{n}^{\prime}\right)=f\left(b_{1}^{\prime}, \ldots, b_{n}^{\prime}\right) \cdot 1$. Since it holds for any $\left(b_{1}^{\prime}, \ldots, b_{n}^{\prime}\right) \in M^{n}$, we get $A_{m}\left[f_{z}\right] \stackrel{m}{=} f_{z}$.

It follows from the theorem that, though applying the $A$-transform to a multiple-valued function $f$ increases the domain of $f$ from $M^{n}$ to $Z_{p}^{n}$, the polynomial $A_{m}[f]$ still yields the same values as $f$ when evaluated for an assignment $\left(b_{1}, \ldots, b_{n}\right) \in M^{n}$. Therefore, the polynomials for two different multiple-valued functions $f$ and $g$ differ on all assignments $\left(b_{1}, \ldots, b_{n}\right) \in M^{n}$ for which $f\left(b_{1}, \ldots, b_{n}\right) \neq g\left(b_{1}, \ldots, b_{n}\right)$. Consequently, $f \neq g$ implies $A_{m}[f] \neq A_{m}[g]$.

\section{Computing the $A$-transform}

Computing $A$-transforms using Definition 3 is feasible for very small functions. For larger functions, we developed an alternative method, which is described in this section.

Let $f_{x_{i}=j}$ denote a subfunction of the function $f\left(x_{1}, \ldots, x_{n}\right)$ with the variable $x_{i}$ being fixed to the value $j$, i.e. $f_{x_{i}=j} \stackrel{d f}{=} f\left(x_{1}, \ldots, x_{i-1}, j, x_{i+1}, \ldots, x_{n}\right)$.

We can apply the following decomposition to a polynomial $A_{m}[f]$, which can be considered as a generalization of Shannon decomposition of Boolean functions:

Theorem 2 Every polynomial $A_{m}[f], m>1$, can be decomposed with respect to a variable $x_{i}$ of $f, i \in\{1, \ldots, n\}$, in the following way

$$
A_{m}[f]=\sum_{j=0}^{m-1}\left(\prod_{k \in M-\{j\}} \frac{k-x_{i}}{k-j}\right) \cdot A_{m}\left[f_{x_{i}=j}\right]
$$

Proof: In order to simplify the exposition and without loss of generality, we show the proof for the case of $x_{i}=x_{1}$. We use $X$ as an abbreviation for $x_{1}, \ldots, x_{n}$.

$$
\begin{aligned}
A_{m} & {[f]=\sum_{\left(b_{1}, . ., b_{n}\right) \in M^{n}} f\left(b_{1}, \ldots, b_{n}\right) \cdot W_{n}\left(b_{1}, \ldots, b_{n}, X\right) } \\
& \{\text { Definition } 3\} \\
= & \sum_{j=0}^{m-1} \sum_{\left(b_{2}, . ., b_{n}\right) \in M^{n-1}} f\left(j, b_{2}, . ., b_{n}\right) \cdot W_{n}\left(j, b_{2}, \ldots, b_{n}, X\right) \\
\{\text { re-grouping }\} & \sum_{j=0}^{m-1} \sum_{\left(b_{2}, \ldots, b_{n}\right) \in M^{n-1}} f\left(j, b_{2}, \ldots, b_{n}\right) \cdot \prod_{k \in M-\{j\}} \frac{k-x_{1}}{k-j} . \\
& \cdot W_{n-1}\left(b_{2}, \ldots, b_{n}, x_{2}, \ldots, x_{n}\right) \\
\left\{w\left(j, x_{1}\right)=\prod_{k \in M-\{j\}} \frac{k-x_{1}}{k-j}, \text { Definition } 2\right\} & \sum_{j=0}^{m-1}\left(\prod_{k \in M-\{j\}} \frac{k-x_{1}}{k-j}\right) \cdot A_{m}\left[f_{x_{1}=j}\right]
\end{aligned}
$$

\{Definition 3\}

Next, we prove a lemma showing how the term $\prod_{k \in M-\{j\}} \frac{k-x}{k-j}, j \in M$, can be expressed by an $m$-equivalent polynomial in linear form.

Lemma 1 For any variable $x$ from $Z_{p}$, any fixed $j \in M$, and $m>2$ :

$$
\prod_{k \in M-\{\}\}} \frac{k-x}{k-j} \stackrel{m}{=}\left\{\begin{array}{r}
\sum_{i=0}^{m-1} a_{i 0} x^{i}, \text { if } j=0 \\
a_{j j} x^{j}+a_{j(m-j)} x^{m-j}, \\
\text { if } j \in M-\{0\} \\
\text { and } j \neq m-j \\
a_{j j} x^{j}, \text { if } j \in M-\{0\} \text { and } j=m-j
\end{array}\right.
$$

where $\forall i, j \in M, a_{i j}=\frac{D_{i j}}{D}$, with $D$ and $D_{i j}$ given by

$$
D=\prod_{i=1}^{m-1} i^{i}-\prod_{i=1}^{m-1} i^{(m-i)}
$$




$$
D_{i j}=\left\{\begin{array}{l}
\prod_{k=1}^{m-1} k^{k \oplus_{m} i}-\prod_{k=1}^{m-1} k^{(m-k) \oplus_{m} i}, \text { if } j=0 \\
\frac{1}{i^{i}} \cdot \prod_{k=1}^{m-1} k^{k}, \text { if } j \neq 0 \text { and } j=i \text { and } j \neq m-i \\
-\frac{1}{(m-i)^{i}} \cdot \prod_{k=1}^{m-1} k^{m-k}, \text { if } j \neq 0 \text { and } j=m-i \\
\text { and } j \neq i \\
\frac{1}{i^{i}} \cdot\left(\prod_{k=1}^{m-1} k^{k}-\prod_{k=1}^{m-1} k^{m-k}\right), \text { if } j \neq 0 \text { and } j=i \\
0, \text { otherwise }
\end{array}\right.
$$

where $" \oplus_{m} "$ denotes addition modulo $m$ and all other operations are regular arithmetic operations in $Z_{p}$.

Proof: We compute the coefficients $a_{i j}, i, j \in M$, by solving the following system of $m$ linear equations with $m$ unknown elements:

$$
\left\{\begin{array}{c}
a_{0 j} \cdot 0^{0}+a_{1 j} \cdot 0^{1}+a_{2 j} \cdot 0^{2}+\ldots+a_{(m-1) j} \cdot 0^{m-1}=b_{0} \\
a_{0 j} \cdot 1^{0}+a_{1 j} \cdot 1^{1}+a_{2 j} \cdot 1^{2}+\ldots+a_{(m-1) j} \cdot 1^{m-1}=b_{1} \\
\ldots \\
a_{0 j} \cdot(m-1)^{0}+a_{1 j} \cdot(m-1)^{1}+a_{2 j} \cdot(m-1)^{2}+\ldots \\
\quad \ldots+a_{(m-1) j} \cdot(m-1)^{m-1}=b_{m-1}
\end{array}\right.
$$

where $\forall i \in M, b_{i}=\prod_{k \in M-\{j\}} \frac{k-i}{k-j}$. Such a system can be described by matrices as $\mathbf{X} \cdot \mathbf{a}=\mathbf{b}$, where

$$
\begin{gathered}
\mathbf{X}=\left(\begin{array}{ccccc}
0^{0} & 0^{1} & 0^{2} & \ldots & 0^{m-1} \\
1^{0} & 1^{1} & 1^{2} & \ldots & 1^{m-1} \\
\ldots & \ldots & \ldots & \ldots & \ldots \\
(m-1)^{0} & (m-1)^{1} & (m-1)^{2} & \ldots & (m-1)^{m-1}
\end{array}\right) \\
\mathbf{a}=\left(\begin{array}{c}
a_{0 j} \\
a_{1 j} \\
\cdots \\
a_{(m-1) j}
\end{array}\right), \mathbf{b}=\left(\begin{array}{c}
b_{0} \\
b_{1} \\
\ldots \\
b_{m-1}
\end{array}\right) .
\end{gathered}
$$

From linear algebra we know that such a system always has a solution, and this solution is unique [10]. We compute the $i$ th element of a by applying Kramer's rule, which says that, for any $i \in M, a_{i j}$ is given by the formula $a_{i j}=\frac{D_{i j}}{D}$, where $D$ is the determinant of $\mathbf{X}$, and $D_{k j}$ is the determinant computed after the replacement of the $i$ th column of $\mathbf{X}$ by vector $\mathbf{b}$.

Observe, that matrix $\mathbf{X}$ has a very regular structure, namely for all $i, j \in M, x_{i j}=i^{j}$. Therefore, by applying standard rules for computing determinants [10], it is easy to show that $D$ and $D_{i j}$ are given by the equation (1).
Examining the structure of $D_{i j}$, we can derive the following properties of the elements of $a_{i j}$ :

$a_{i j}= \begin{cases}\frac{D_{i j}}{D} & \forall i, j: \text { such that } j=0 \text { or } i=j \text { or } i=m-j \\ 0 & \text { otherwise }\end{cases}$

So, the only elements $a_{i j}$ which can possibly have nonzero values are $a_{i 0}$ for all $i \in M$, and $a_{j j}$ and $a_{j(m-j)}$ for all $j \in M-\{0\}$. Therefore, the expression for the term

$\prod_{k \in M-\{j\}} \frac{k-x}{k-j}$ can be simplified to:

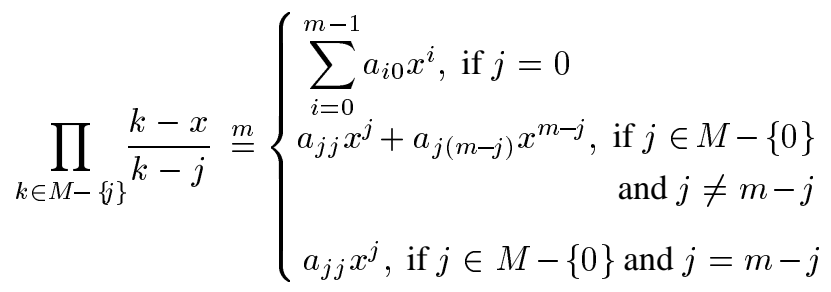

As we mentioned above, $\prod_{k \in M-\{\}\}} \frac{k-x}{k-j}$ has a behavior similar to the behavior of the literal operator $\stackrel{j}{x}$, except that for $x=j$ the literal $\stackrel{j}{x}$ evaluates to $m-1$, and not to 1 . Therefore, $\stackrel{j}{x} \stackrel{m}{=}(m-1) \cdot \prod_{k \in M-\{\}} \frac{k-x}{k-j}$, and thus, from Lemma 1, we can conclude that

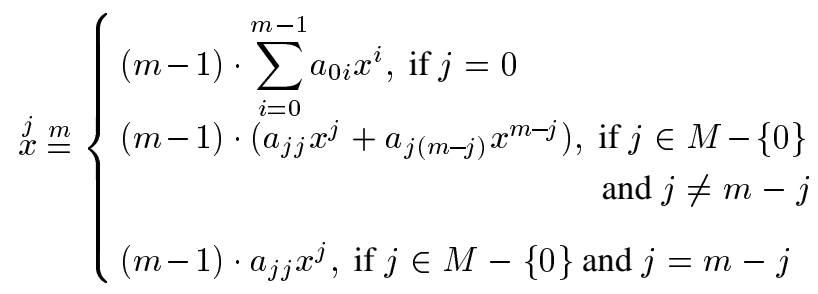

We use Theorem 2 and Lemma 1 to derive another type of decomposition of $A_{m}[f]$, which will be used later to derive a canonical expansion for $A_{m}[f]$. Before giving the decomposition, we first summarize some properties of $A_{m}[f]$, obvious from Definition 3.

Lemma 2 For any field functions $f_{z}$ and $g_{z}$ and any constant $c \in Z_{p}$,

(a) $A_{m}\left[c \cdot f_{z}\right]=c \cdot A_{m}\left[f_{z}\right]$.

(b) $A_{m}\left[f_{z}+g_{z}\right]=A_{m}\left[f_{z}\right]+A_{m}\left[g_{z}\right]$.

Theorem 3 Every polynomial $A_{m}[f], m>1$, can be decomposed with respect to a variable $x$ of $f$ in the following way:

case 1: if $m$ is odd, then

$$
\begin{gathered}
A_{m}[f]=a_{00} A_{m}\left[f_{x=0}\right]+\sum_{j=1}^{m-1}\left(\left(a_{j 0} A_{m}\left[f_{x=0}\right]+\right.\right. \\
\left.\left.+a_{j j} A_{m}\left[f_{x=j}\right]+a_{j(m-j)} A_{m}\left[f_{x=m-j}\right]\right) \cdot x^{j}\right)
\end{gathered}
$$


case 2: if $m$ is even, then

$$
\begin{aligned}
& A_{m}[f]=a_{00} A_{m}\left[f_{x=0}\right]+\sum_{\substack{j=1 \\
j \neq m / 2}}^{m-1}\left(\left(a_{j 0} A_{m}\left[f_{x=0}\right]+\right.\right. \\
& \left.+a_{j j} A_{m}\left[f_{x=j}\right]+a_{j(m-j)} A_{m}\left[f_{x=m-j}\right]\right) \cdot x^{j}+ \\
& \left.+\left(a_{\frac{m}{2} 0} A_{m}\left[f_{x=0}\right]+a_{\frac{m}{2} \frac{m}{2}} A_{m}\left[f_{x=m / 2}\right]\right) \cdot x^{m / 2}\right)
\end{aligned}
$$

where $\forall i, j \in M, a_{i j}=\frac{D_{i j}}{D}$, and $D$ and $D_{i j}$ given by (1).

Proof: $A_{m}[f]=$

$$
\begin{aligned}
& =\sum_{j=0}^{m-1}\left(\prod_{k \in M-\{j\}} \frac{k-x_{i}}{k-j}\right) \cdot A_{m}\left[f_{x_{i}=k}\right] \quad\{\text { Theorem } 2\} \\
& =\sum_{i=0}^{m-1} a_{i 0} x^{i} \cdot A_{m}\left[f_{x=0}\right]+\sum_{j=1}^{m-1}\left(a_{j j} x^{j}+a_{j(m-j)} x^{m-j}\right) . \\
& \cdot A_{m}\left[f_{x=j}\right] \\
& =a_{00} A_{m}\left[f_{x=0}\right]+\sum_{j=1}^{m-1}\left(a_{j 0} A_{m}\left[f_{x=0}\right]+a_{j j} A_{m}\left[f_{x=j}\right]+\right. \\
& \left.+a_{j(m-j)} A_{m}\left[f_{x=m-j}\right]\right) \cdot x^{j} \quad\{\text { reordering }\}
\end{aligned}
$$

Let $F$ be the vector of coefficients of the truth table of the function $f$ and $A^{n}$ be a transformation matrix, defined as follows:

Definition 5 The $m^{n} \times m^{n}$ matrix $A^{n}$ is defined inductively by:

\section{1. $A^{1} \stackrel{d f}{=}$}

$$
\left[\begin{array}{ccccc}
a_{00} & 0 & 0 & \ldots & 0 \\
a_{10} & a_{11} & 0 & \ldots & a_{1(m-1)} \\
a_{20} & 0 & a_{22} & \ldots & 0 \\
\ldots & \ldots & \ldots & \ldots & \\
a_{(m-2) 0} & 0 & a_{(m-2) 2} & \ldots & 0 \\
a_{(m-1) 0} & a_{(m-1) 1} & 0 & \ldots & a_{(m-1)(m-1)}
\end{array}\right]
$$

where $\forall i, j \in M$, the coefficients $a_{i j}$ are given by (2).

$$
\text { 2. } A^{n} \stackrel{d f}{=} A^{1} \otimes A^{n-1}
$$

where" $\otimes "$ denotes the Kronecker product of two matrices.

Clearly, if Theorem 3 is successively applied to the polynomials $A_{m}\left[f_{x_{i}=k}\right]$ of subfunctions $f_{x_{i}=k}$ about all the remaining variables, we will finally get an expression in which $A_{m}[f]$ is expanded in all the variables of $f$ :
Theorem 4 Every polynomial $A_{m}[f], m>2$, of an $n$ variable $m$-valued function $f$ can be expressed in the following canonical form

$$
A_{m}[f]=\sum_{j=0}^{m^{n}-1} c_{j} \cdot x_{1}^{i_{1}} \cdot x_{2}^{i_{2}} \cdot \ldots \cdot x_{n}^{i_{n}}
$$

where $\left(i_{1} i_{2} \ldots i_{n}\right)$ is the $m$-ary expansion of $i$, with $i_{1}$ being the least significant digit, and the coefficients $c_{i}$ are given by the vector $C \stackrel{d f}{=}\left[c_{0} c_{1} \ldots c_{m^{n}-1}\right]$ computed as $C=A^{n} \cdot F$.

Proof: By induction on $n$. We show the proof only for the case of $m$ being odd. For $m$ - even the proof is similar.

1) Let $n=1$. According to Theorem 3, any polynomial $A_{m}[f]$ of a function $f(x)$ of one variable $x$ can be decomposed with respect to $x$ as:

$$
\begin{aligned}
& A_{m}[f]=a_{00} \cdot A_{m}\left[f_{x=0}\right]+\sum_{j=1}^{m-1}\left(a_{j 0} \cdot A_{m}\left[f_{x=0}\right]+\right. \\
& \left.+a_{j j} \cdot A_{m}\left[f_{x=j}\right]+a_{j(m-j)} \cdot A\left[f_{x=m-j}\right]\right) \cdot x^{j}
\end{aligned}
$$

where $f_{x=k}=f(k)$. By Lemma $2, A_{m}[c]=c$ for any constant $c \in Z_{p}$. So, we can express the above as:

$$
\begin{aligned}
& A_{m}[f]=a_{0} 0 \cdot f(0)+\sum_{j=1}^{m-1}\left(a_{j 0} \cdot f(0)+a_{j j} \cdot f(j)+\right. \\
& \left.+a_{j(m-j)} \cdot f(m-j)\right) \cdot x^{j}
\end{aligned}
$$

which can be re-written as

$$
A_{m}[f]=\sum_{i=0}^{m-1} c_{i} x^{i}
$$

where $c_{0}=a_{00} \cdot f(0)$ and $c_{j}=a_{j 0} \cdot f(0)+a_{j j} \cdot f(j)+$ $a_{j(m-j)} \cdot f(m-j)$, for all $j \in M-\{0\}$. Examining the structure of the matrix $A^{1}$, we can conclude that $C=A^{1} \cdot F$.

2) Hypothesis: Assume the result for $n$. According to Theorem 3 , any $A_{m}[f]$ of a function $f$ of $n+1$ variables can be decomposed with respect to $x_{n+1}$ in the following way:

$$
\begin{aligned}
& A_{m}[f]=a_{00} A_{m}\left[f_{x_{n+1}=0}\right]+\sum_{j=1}^{m-1}\left(a_{j 0} A_{m}\left[f_{x=0}\right]+\right. \\
& \left.\quad+a_{j j} A_{m}\left[f_{x=j}\right]+a_{j(m-j)} A_{m}\left[f_{x=m-j}\right]\right) \cdot x_{n+1}^{j}
\end{aligned}
$$

By the induction hypothesis, we can express each $A_{m}$ of the subfunctions of $n$ variables in the canonical form. We use the notation $c_{i}^{k}$ to denote the $i$ th coefficient of the canonical form of the subfunction $f_{x_{n+1}=k}$ and $F_{k}$ for the truth table vector of $f_{x_{n+1}=k}$. To simplify the exposition we also use the abbreviation $X$ to stand for the term $x_{1}^{i_{1}} \cdot x_{2}^{i_{2}} \cdot \ldots \cdot x_{n}^{i_{n}}$. 
So, we replace each of $A_{m}\left[f_{x_{n+1}=k}\right], k \in M$, by $\sum_{i=0}^{m-1} c_{i}^{k} \cdot X$, with $c_{i}^{k}$ given by $C_{k}=A^{n} \cdot F_{k}$. Then we get:

$$
\begin{aligned}
& A_{m}[f]=a_{00} \cdot \sum_{i=0}^{m^{n}-1} c_{i}^{0} \cdot X+\sum_{j=1}^{m-1}\left(a_{j 0} \cdot \sum_{i=0}^{m^{n}-1} c_{i}^{0} \cdot X+\right. \\
& \left.+a_{j j} \cdot \sum_{i=0}^{n-1} c_{i}^{j} \cdot X+a_{j(m-j)} \cdot \sum_{i=0}^{m^{n}-1} c_{i}^{m-j} \cdot X\right) \cdot x_{n+1}^{j}
\end{aligned}
$$

Since ". " is distributive over " +", we can re-order the above as

$$
\begin{aligned}
& A_{m}[f]=\left(\sum_{i=0}^{m^{n}-1} a_{00} \cdot c_{i}^{0} \cdot X\right) \cdot x_{n+1}^{0}+\sum_{j=1}^{m-1}\left(\sum _ { i = 0 } ^ { m ^ { n } - 1 } \left(a_{j 0} \cdot\right.\right. \\
& \left.\left.\cdot c_{i}^{0}+a_{j j} \cdot c_{i}^{j}+a_{j(m-j)} c_{i}^{m-j}\right) \cdot X\right) \cdot x_{n+1}^{j}
\end{aligned}
$$

which can be re-written as

$$
A_{m}[f]=\sum_{j=0}^{m^{n}-1} c_{j} \cdot x_{1}^{i_{1}} \cdot x_{2}^{i_{2}} \cdot \ldots \cdot x_{n}^{i_{n}}
$$

where $c_{i}=a_{00} \cdot c_{i}^{0}$, for $0 \leq i \leq m-1$, and $c_{i}=a_{j 0} \cdot c_{i}^{0}+$ $a_{j j} \cdot c_{i}^{j}+a_{j(m-j)} c_{i}^{m-j}$, for $j \cdot m \leq i \leq j \cdot m+m-1$, for all $j \in M-\{0\}$. Since the coefficients $c_{i}^{j}$ are given by $C_{j}=$ $A^{n} \cdot F_{j}$, this is equivalent to $C=\left(A^{1} \otimes A^{n}\right) \cdot F=A^{n+1} \cdot F$.

For example, for $m=3$ and $n=2$, the matrix $A^{2}$ is constructed as follows:

$$
A^{1}=\left[\begin{array}{rrr}
1 & 0 & 0 \\
-\frac{3}{2} & 2 & -\frac{1}{2} \\
\frac{1}{2} & -1 & \frac{1}{2}
\end{array}\right]
$$

and $A^{2}=$

$$
\left[\begin{array}{rrrrrrrrr}
1 & 0 & 0 & 0 & 0 & 0 & 0 & 0 & 0 \\
-\frac{3}{2} & 2 & -\frac{1}{2} & 0 & 0 & 0 & 0 & 0 & 0 \\
\frac{1}{2} & -1 & \frac{1}{2} & 0 & 0 & 0 & 0 & 0 & 0 \\
-\frac{3}{2} & 0 & 0 & 2 & 0 & 0 & -\frac{1}{2} & 0 & 0 \\
\frac{9}{4} & -3 & \frac{3}{4} & -3 & 4 & -1 & \frac{3}{4} & -1 & \frac{1}{4} \\
-\frac{3}{4} & \frac{3}{2} & -\frac{3}{4} & 1 & -2 & 1 & -\frac{1}{4} & \frac{1}{2} & -\frac{1}{4} \\
\frac{1}{2} & 0 & 0 & -1 & 0 & 0 & \frac{1}{2} & 0 & 0 \\
-\frac{3}{4} & 1 & -\frac{1}{4} & \frac{3}{2} & -2 & \frac{1}{2} & -\frac{3}{4} & 1 & -\frac{1}{4} \\
\frac{1}{4} & -\frac{1}{2} & \frac{1}{4} & -\frac{1}{2} & 1 & -\frac{1}{2} & \frac{1}{4} & -\frac{1}{2} & \frac{1}{4}
\end{array}\right]
$$

So, we can compute $A_{3}[f]$ for $f=\operatorname{MIN}\left(x_{1}, x_{2}\right)$ with $F=$

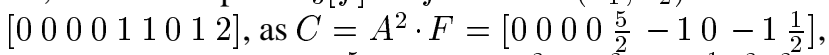
giving $A_{3}[f]\left(x_{1}, x_{2}\right)=\frac{5}{2} x_{1} x_{2}-x_{1} x_{1}^{2}-x_{1}^{2} x_{2}+\frac{1}{2} x_{1}^{2} x_{2}^{2}$.

\section{Computing hash code of the function}

We compute the hash code of the function $f$ by assigning randomly chosen integer values from $Z_{p}$ to the input variables of $f$, and then evaluating $A_{m}[f]$ for this values. The resulting number is the integer hash code of $f$. This code requires less space than the canonical MDD representation of the same function and distinguishes any pair of multiple-valued functions with a quantifiable probability of success. The hash codes for two equivalent functions are always the same. Thus, the equivalence of two functions can be verified with a known probability of error, which arises from collisions between inequivalent functions. According to Schwartz-Zippel Theorem [11, p. 165], if the assignments of values of variables $x_{1}, \ldots, x_{n}$ are taken independently and uniformly at random from a field $\mathcal{F}$ of size $|\mathcal{F}|$, then the hash codes for two equivalent functions can be distinguished with the probability at least $\frac{n}{|\mathcal{F}|}$. In our case $\mathcal{F}=Z_{p}$ and $|\mathcal{F}|=p$, so we get $\frac{n}{p}$. A smaller error bound $\left(\frac{p-1}{p}\right)^{n}$ is derived in [9].

As an example, consider the 3-valued 2-variable function $f=\operatorname{MIN}\left(x_{1}, x_{2}\right)$ and let the variables be assigned the random values $x_{1}=2, x_{2}=4$. Since $A_{3}[f]=\frac{5}{2} x_{1} x_{2}-$ $x_{1} x_{1}^{2}-x_{1}^{2} x_{2}+\frac{1}{2} x_{1}^{2} x_{2}^{2}$, the hash code for $f$ is 4 .

As another example, consider the function $g=$ $\operatorname{MAX}\left(\operatorname{MIN}\left(2, \stackrel{2}{x_{1}}, \stackrel{0}{x_{2}}\right), \operatorname{MIN}\left(1, \stackrel{2}{x_{1}}, \stackrel{1}{x}_{2}\right)\right)$. Since $A_{3}[g]=$ $-2 x_{1}+2 x_{1} x_{2}-\frac{1}{2} x_{1} x_{2}^{2}+2 x_{1}^{2}-2 x_{1}^{2} x_{2}+\frac{1}{2} x_{1}^{2} x_{2}^{2}$, if we assign $x_{1}=2$ and $x_{2}=4$ we obtain the same hash code 4 as for MIN function and therefore get collision between two inequivalent functions.

However, we can substantially decrease the probability of collision by making multiple runs. On each run, an independent set of input variable assignment is randomly chosen, and the two function values are computed. If the values differ, we are assured that the two functions are not the same. If they are equal, we choose a new set of input assignments and re-evaluate. The probability of incorrectly deciding that the functions are equal decrease exponentially with the number of runs: if the error probability of a single run is $e$, then after $k$ runs the error probability is $e^{k}$ [12]. For example, if we make a second run for the functions specified above, with the random values $x_{1}=3$ and $x_{2}=1$, then the hash code for $M I N$ function is 0 and hash code for $g$ function is 3 . So, we can conclude that $f \neq g$.

\section{Conclusion}

This paper lays a theoretical foundation of a probabilistic method for verification of multiple-valued functions. We define a functional transformation, which is can be used to obtain an integer code from a multiple-valued function. 
The integer codes allow us to determine probabilistically whether two functions are equivalent.

Further research remains designing an efficient procedure for computing integer hash codes. A simple way to compute a hash code would be to build an MDD or similar structure from the input function and then apply a procedure for reducing this structure to an appropriate integer. Of course, the efficiency of such a scheme would be limited by the need to create and evaluate an MDD representation of the entire function. A better approach, which we are currently pursuing, is first to symbolically decompose the function, and then hash it incrementally. This can be done by first hashing some of its parts, and then using these more compact intermediate forms to complete the hashing of the entire function.

\section{References}

[1] A. Nozoe et al., A 256-Mb multilevel flash memory with $2 \mathrm{MB} / \mathrm{s}$ program rate for mass storage applications, Proc. of 1999 IEEE Int. Solid-State Circuits Conference (ISSCC'99), (1999), 110-111.

[2] T. Okuda, T. Murotani, A four-level storage 4-Gb DRAM IEEE Journal of Solid-State Circuits 32, 11, (1997), 1743 - 1747.

[3] The VIS Group, VIS: A system for verification and synthesis, Proc. 8th Int. Conf. on Computer Aided Verification, Springer Lecture Notes in Computer Science, 1102, Edited by R. Alur and T. Henzinger, New Brunswick, NJ, (1996), 428-432.

[4] R. Drechsler, M. Keim, B. Becker, Fault simulation for sequential multi-valued logic networks, Proc. 27th Int. Symp. on Multiple-Valued Logic (1997), 145-150.

[5] R. E. Bryant, C.-J. H. Seger, Digital circuit verification using partially-ordered state models, Proc. 24th Int. Symp. on Multiple-Valued Logic (1994), 2-7.

[6] R.E. Bryant, Graph-based algorithm for Boolean function manipulation, IEEE Transactions on Computers C-35 No. 8 (1986), 677-691.

[7] D. M. Miller, Multiple-valued logic design tools, Proc. 23rd Int. Symp. on Multiple-Valued Logic (1993), 2-11.

[8] R. Drechsler, Verification of multiple-valued logic networks, Proc. 26th Int. Symp. on Multiple-Valued Logic (1996), 10-15.

[9] J. Jain, J. Bitner, D. S. Fussell, J. A. Abraham, Probabilistic verification of Boolean functions, Formal Methods in System Design, Kluwer Academic Publishers, 1, (1992), 63-117.
[10] G. Birkhoff, S. MacLane, Brief Survey of Modern Algebra, 4th ed., New York, Macmillan, 1977.

[11] R. Motwani, P. Raghavan, Randomized Algorithms, Cambridge University Press, 1995.

[12] M. Blum, A. K. Chandra, M. N. Wegman, Equivalence of free Boolean graphs can be decided probabilistically in polynomial time, Information Processing letters 10, No. 2, (1980), 80-82. 